\title{
Produção, caracterização e avaliação da capacidade adsortiva de carvões ativados em forma de briquete
}

\author{
Production, characterization and evaluation \\ of the adsorptive potential of activated \\ carbon briquette shaped
}

\author{
Willian Miguel da Silva Borges ${ }^{1}$, Ana Carolina Cunha Aran- \\ tes ${ }^{1}$,Guilherme Mello Mattos de Castro ${ }^{1}$, Maria Lucia Bianchi ${ }^{1}$, \\ João Rodrigo Coimbra Nobre ${ }^{2}$, Eliane Cristina de Resende ${ }^{3}$, \\ Jonnys Paz Castro ${ }^{1}$, Mário César Guerreiro ${ }^{1}$
}

\begin{abstract}
${ }^{1}$ Universidade Federal de Lavras, Departamento de Química, CEP 37200-000, Lavras-MG, Brasile-mail: will_msb@hotmail.com,anacarolinacarantes@gmail.com,g.mmcastro@hotmail.com,bianchi@dqi.ufla.br, jonnys_33@hotmail.com, guerreiro@dqi.ufla.br.

${ }^{2}$ Universidade do Estado do Pará, Centro de Ciências Naturais e Tecnologia,Departamento de Tecnologia e Recursos Naturais. Campus Universitário - 68628-557 - Paragominas, PA. e-mail: rodrigonobre@hotmail.com.br ${ }^{3}$ Instituto Federal de Minas Gerais - Campus Bambuí - Caixa Postal 05 - CEP: 38900-000. e-mail: eliane.resende@ifmg.edu.br
\end{abstract}

\section{RESUMO}

Carvões ativados em forma de briquetes foram preparados a partir do resíduo da madeira de candeia, gerado no processo de extração do óleo $\alpha$-bisabolol. O material foi briquetado, carbonizado e ativado com $\mathrm{CO}_{2}$, variando-se a temperatura $\left(700-900^{\circ} \mathrm{C}\right)$ e o tempo de ativação (1-3 horas). Para a caracterização dos carvões ativados foram realizadas análise elementar (CHN-O), análise dos grupos funcionais (titulação de Boehm), buroff, área superficial BET estimada, índice de iodo, cálculo de área superficial com azul de metileno $\mathrm{S}_{\mathrm{AM}}$, testes de adsorção com azul de metileno e fenol e índice de resistência mecânica. Os resultados mostraram que, com o aumento da temperatura e do tempo de ativação, ocorre aumento da área superficial, do volume de poros, da basicidade do carvão e da capacidade de adsorção de azul de metileno e de fenol.

Palavras-chave:Candeia; Carvão Ativado; Briquete.

\section{ABSTRACT}

Activated carbons briquette shaped were prepared from Candeia wood waste, generated in the extraction of $\alpha$-bisabololoil process. The material was briquetted, carbonized and activated with $\mathrm{CO}_{2}$, varying the temperature $\left(700-900^{\circ} \mathrm{C}\right)$ and activation time (1-3 hours). The characterization of the activated carbons were performed by: elemental analysis (CHN-O), analysis of the functional groups (Boehm titration), Burn-off, estimated BET surface area, iodine index, calculation of surface area with methylene blue, adsorption tests with methylene blue (MB) and phenol and mechanical resistance (IRI). The results showed that increasing temperature and time of activation promoted an increased in the surface area, pore volume, basicity and adsorption capacity of AM and phenol.

Keywords: Candeia; Activated charcoal; Briquette

\section{INTRODUÇÃO}

A indústria de extração do óleo $\alpha$-bisabolol a partir da madeira de candeia gera grande volume de resíduo. $O$ óleo extraído a partir da candeia é utilizado na indústria farmacêutica e de cosméticos. Parte do resíduo é utilizada para fornecer energia para as caldeiras durante o processo de extração e purificação do óleo; outra parte é acumulada nos pátios das fábricas, o que gera problemas com armazenamento e contaminação do ambiente [1]. Resíduos de candeia gerados a partir da produção de $\alpha$-bisabolol são fontes ricas em carbono. Uma utilização alternativa para este material é a preparação de carvão ativado [2]. 
Carvões ativados são materiais carbonáceos caracterizados pela elevada área superficial e estrutura porosa altamente desenvolvida [3]. A escolha do precursor e as condições de ativação nos dão a possibilidade de conceber carvões ativados para diferentes aplicações. Atualmente, eles são amplamente utilizados em uma gama de aplicações, tais como usos médicos, remoção de poluentes e odores, separação de gases, suporte para catalisadores e purificação de água, dentre outros [4].

Dois tipos de processos são utilizados para produzir carvão ativado, a ativação química e a ativação física. Na ativação química, o material bruto, normalmente, é ativado por agentes ativadores, tais como $\mathrm{KOH}$,

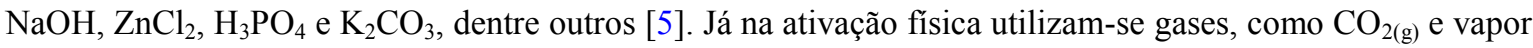
de água no processo de tratamento do material. Entretanto, o processo químico de ativação gera uma grande quantidade de resíduo e, portanto, provoca um maior impacto ambiental além de ser mais oneroso. Já no processo físico, são utilizadas temperaturas elevadas, porém, há uma menor geração de resíduos. Dessa forma, o impacto ambiental do processo físico é menor [6,7].

Carvões ativados podem ser trabalhados em forma de briquetes [8], pó ou granular, podendo ser utilizado em equipamentos como purificadores de água que trabalham com baixas taxas de fluxo de fluidos (isto é, ar ou água) em contato com o carvão ativado. Quando na forma de pó podem espalhar-se no recipiente, sendo arrastados para o interior do equipamento, causando problemas mecânicos nos casos em que a taxa do fluxo do fluido é relativamente alta, tal como num filtro de um aparelho de ar condicionado. Assim, os grânulos duros, pellets ou briquetes são utilizados em processos contínuos ou cíclicos, em que a força mecânica é uma propriedade importante a ser considerada [9].

Para o processo de briquetagem geralmente são utilizados ligantes, para que os briquetes ganhem resistência mecânica. Porém trabalhos realizados mostram que na produção de briquetes para utilização como material precursor na produção de carvão ativado não é necessário o uso de um material adesivo ou colante e pode-se utilizar somente o material lignocelulósico [10]. O aumento da temperatura faz com que a lignina se torne plástica e atue como ligante natural das partículas durante a compactação. Além da lignina, proteínas, amido, gorduras e carboidratos também são adesivos naturais da biomassa [11].

O presente trabalho teve como objetivo produzir carvões ativados em forma de briquete (CAB) a partir do resíduo madeireiro gerado da extração do óleo de candeia, utilizando o processo de ativação física variando-se a temperatura e o tempo de ativação. Os materiais obtidos foram caracterizados e avaliados quanto à capacidade de adsorção de azul de metileno e fenol.

\section{MATERIAIS E MÉTODOS}

\subsection{Amostras}

O resíduo madeireiro da candeia (Eremanthuserythropappus (DC.) MacLeish), utilizado como precursor na produção dos carvões ativados, foi cedido pela empresa Atina - Ativos Naturais - Ltda. (Pouso Alegre, MG).

Os principais constituintes do resíduo estão descritos na Tabela 1. O baixo teor de cinzas e a quantidade de lignina presente no material são características do resíduo de candeia que fazem dele um bom material precursor para a produção de carvão ativado $[4,12]$.

Tabela 1: Constituição do resíduo de Candeia

\begin{tabular}{c|c}
\hline \multicolumn{2}{c}{ Características (\%) } \\
\hline Cinza & 1,1 \\
\hline Extrativo & 12,1 \\
\hline Lignina insolúvel & 32,0 \\
\hline Holocelulose & 57,3 \\
\hline
\end{tabular}

\subsection{Briquetagem}

O resíduo madeireiro da candeia utilizado na briquetagem foi peneirado em peneiras com granulometria de 60 mesh. Foi empregada uma pressão de 150 bar para a produção dos briquetes $\left(153 \mathrm{kgf} \mathrm{cm}^{-2}\right)$, à temperatura de $150{ }^{\circ} \mathrm{C}$ e tempo de residência de 8 minutos. A quantidade de material inserido na briquetadeira para a produção de cada briquete foi de $6 \mathrm{~g}$. 


\subsection{Carbonização}

O material precursor briquetado foi carbonizado em forno tipo mufla. Aproximadamente 25 briquetes foram inseridos em uma cápsula de aço inox (reator) e, posteriormente, colocados dentro da mufla. A mufla foi aquecida até uma temperatura de $40{ }^{\circ} \mathrm{C}$, com uma taxa de aquecimento de $1,67^{\circ} \mathrm{C} \mathrm{min}^{-1}$ permanecendo 30 minutos nessa temperatura. Em seguida, com a mesma taxa de aquecimento, elevou-se a temperatura até $550{ }^{\circ} \mathrm{C}$ mantendo essa temperatura por 1 hora. O resfriamento ocorreu de forma natural e gradativa após o forno atingir a temperatura final de carbonização.

\subsection{Processo de ativação dos carvões}

$\mathrm{O}$ material carbonizado foi ativado em forno tubular elétrico utilizando-se $\mathrm{CO}_{2(\mathrm{~g})}$ como agente oxidante. Em cada processo de ativação utilizaram-se 7 briquetes. O tubo utilizado na ativação dos materiais é de molita 710 , com medidas de 46,5 mm de diâmetro externo, 3,5 $\mathrm{mm}$ de espessura e $80 \mathrm{~cm}$ de comprimento. Depois dos materiais terem sido inseridos no tubo, o mesmo foi levado para dentro de um forno tubular e fechado nas extremidades com uma rolha de borracha furada para permitir o fluxo de $\mathrm{CO}_{2(\mathrm{~g})}$ no interior do tubo. Um rotâmetro foi utilizado para a regulagem do fluxo de $\mathrm{CO}_{2(\mathrm{~g})}$.

Os carvões briquetados e carbonizados foram ativados nas temperaturas de $700{ }^{\circ} \mathrm{C}, 800{ }^{\circ} \mathrm{C}$ e $900{ }^{\circ} \mathrm{C}$ e tempo de permanência, à temperatura máxima, de 1 e 3 horas. Os materiais foram denominados conforme a temperatura máxima e tempo de permanência CAB700-1, CAB700-3, CAB800-1, CAB800-3, CAB900-1 e CAB900-3.

O fluxo de $\mathrm{CO}_{2(\mathrm{~g})}$ utilizado foi de $100 \mathrm{~mL} \mathrm{~min}^{-1}$, para todas as ativações. A rampa de aquecimento utilizada foi de $10^{\circ} \mathrm{C} \mathrm{min}^{-1}$, até a temperatura máxima. Ao final do processo, o forno foi desligado para que ocorresse o resfriamento gradativo do material. O fluxo de $\mathrm{CO}_{2(\mathrm{~g})}$ foi mantido até a temperatura de $200{ }^{\circ} \mathrm{C}$.

\subsection{Caracterização dos materiais}

A quantificação dos teores de carbono, hidrogênio e nitrogênio $(\mathrm{CHN})$ foi realizada por meio de um analisador simultâneo de elementos (modelo Vario Micro cube, fabricado na Alemanha pela empresa ELEMENTAR). $O$ teor de oxigênio foi determinado por diferença.

O método de Boehm foi utilizado para determinar os grupos químicos presentes na superfície dos carvões ativados em forma de briquetes [13]. Foi utilizado um titulador potenciométrico Metrohm (modelo Titrando 888, fabricado na Suíça). A metodologia baseia-se no uso de reações da química orgânica para a caracterização dos oxigênios quimiossorvidos na superfície do carvão ativado, como grupos carboxílicos, fenólicos e lactonas $[13,14]$. O número de sítios com caráter básico pode ser avaliado utilizando-se HCl. OsCABs foram macerados para a realização do procedimento que consistiu em pesar, aproximadamente, 0,25 $\mathrm{g}$ de material que foram, posteriormente, colocados sob agitação, durante 24 horas, com $10 \mathrm{~mL}$ de $\mathrm{NaHCO}_{3} 0,05$ mol L $\mathrm{L}^{-1}$. O mesmo procedimento foi realizado utilizando-se os reagentes $\mathrm{Na}_{2} \mathrm{CO}_{3}, \mathrm{NaOH}$ e $\mathrm{HCl}\left(0,05 \mathrm{~mol} \mathrm{~L}^{-}\right.$ $\left.{ }^{1}\right)$. Após esse período, os carvões foram filtrados em papel de filtro $\left(80 \mathrm{gm}^{-2}\right.$ de gramatura, $205 \mu \mathrm{m}$ de espessura e $14 \mu \mathrm{m}$ de poros) e uma alíquota de $5 \mathrm{~mL}$ foi retirada de cada frasco. Nas amostras preparadas com $\mathrm{NaOH}$ e $\mathrm{NaHCO}_{3}$, adicionaram-se $10 \mathrm{~mL}$ de $\mathrm{HCl} 0,05$ mol L ${ }^{-1}$ na amostra preparada com $\mathrm{Na}_{2} \mathrm{CO}_{3}$, adicionaram-se $15 \mathrm{~mL}$ de $\mathrm{HCl}$ 0,05 $\mathrm{mol} \mathrm{L}^{-1} \mathrm{e}$, na amostra preparada com $\mathrm{HCl}$, adicionaram-se $10 \mathrm{~mL}$ de $\mathrm{NaOH}$. Essa mistura foi deixada durante duas horas na presença de fluxo de $\mathrm{N}_{2(\mathrm{~g})}$, para total desgaseificação. Após as duas horas, realizou-se a titulação, em um titulador automático, com solução padronizada de $\mathrm{NaOH}$ ou $\mathrm{HCl}$. A partir destes dados pode-se calcular o número de sítios relacionados ao conjunto de grupos orgânicos de caráter ácido e básico $[15,16]$.

Foi calculada a redução de massa das amostras (grau de burn-off) e o rendimento, em massa, do material carbonizado e ativado nas diferentes temperaturas e tempos, conforme as Equações 1 e 2.

$$
\begin{aligned}
& \text { Cálculodoburn }- \text { offBO }=\frac{M_{\text {Inicial }}-M_{\text {Final }}}{M_{\text {Inicial }}} \times 100 \\
& \text { Rendimento } \%=\frac{\mathrm{M}_{\text {Final }}}{\mathrm{M}_{\text {Inicial }}} \times 100
\end{aligned}
$$

Onde para o cálculo de burn-off, $\mathrm{M}_{\text {inicial }}$ é a massa inicial do material já carbonizado pronto para a ativação e $\mathrm{M}_{\text {final }}$ é a massa obtida após a ativação. 


\subsection{Cálculo da área BET estimada}

A área BET descreve a adsorção física de moléculas de gás sobre uma superfície sólida, e pode servir como base para a técnica de análise de medição de área superficial específica de um material [17].

Para o cálculo da área da superfície, o volume de microporos e o volume total de poros de carvões ativados foi utilizado o método desenvolvido por NUNES e GUERREIRO [18], que consiste em demonstrar que a área de superfície, o volume de microporos e o volume total de poros do carvão ativado podem ser estimados utilizando-se os valores de números de iodo e capacidade de adsorção de azul de metileno por meio de regressão múltipla. Foi empregado o software "Structural Characterization of Activated Carbon" (Desenvolvido na Universidade Federal de Lavras, Brasil), para calcular a área de superfície, o volume de microporos e o volume de poros total, utilizando os modelos desenvolvidos [18].

\section{7 Índice de lodo e cálculo de área superficial com azul de metileno $\mathrm{S}_{\mathrm{AM}}$}

O índice de iodo foi calculado segundo a norma descrito no Standard Test Method (D 4607-94) [19], em que o índice de iodo é definido como a quantidade $(\mathrm{mg})$ de iodo adsorvida por grama de adsorvente.

Uma forma de se estimar a área superficial do carvão ativado é por meio da molécula modelo de azul de metileno. A molécula do corante catiônico azul de metileno, apresentada na Figura 1 é, geralmente, empregada como adsorbato, em testes de adsorção em fase líquida.

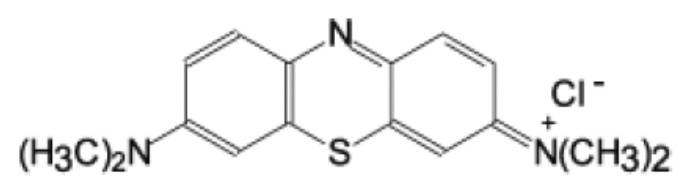

Figura 1: Molécula de azul de metileno

Por meio da área superficial do azul de metileno é possível estimar a área do carvão ativado pela Equação 3.

$$
S_{A M}=1000 * S_{A M}^{\circ} * q m
$$

em que $\mathrm{S}_{\mathrm{AM}}\left(\mathrm{m}^{2} \mathrm{~g}^{-1}\right)$ é a área do carvão ativado acessível ao azul de metileno, $\mathrm{S}_{\mathrm{AM}}^{\circ}$ é a área superficial do azul de metileno $\left(1,93 \mathrm{~m}^{2} \mathrm{mg}^{-1}\right)$ e $\mathrm{q}_{\mathrm{m}}\left(\mathrm{mg} \mathrm{g}^{-1}\right)$ é a capacidade máxima de adsorção de azul de metileno no carvão ativado [20].

\subsection{Teste de adsorção}

O procedimento feito em batelada consiste em colocar um briquete ativado em contato com $100 \mathrm{~mL}$ de solução do adsorbato azul de metileno ou fenol em diferentes concentrações, variando de 25 a $1000 \mathrm{mg} \mathrm{L}^{-1}$. Os frascos foram mantidos sob agitação por 24 horas, à temperatura de $25 \pm 1^{\circ} \mathrm{C}$. Em seguida, a concentração remanescente foi monitorada por espectroscopia de UV-Visível, nos comprimentos de onda correspondentes AM: $\lambda=665 \mathrm{~nm}$ e fenol: $\lambda=270 \mathrm{~nm}$. Os dados das isotermas de equilíbrio foram ajustados segundo os modelos de Langmuir e Freundlich, Equações 4 e 5, em que $\mathrm{q}_{\mathrm{m}}$ é a capacidade máxima de adsorção; $\mathrm{K}_{\mathrm{L}} \mathrm{e}$ a constante de Langmuir; $\mathrm{K}_{\mathrm{F}}$ e $\mathrm{n}$ são os coeficientes de Freundlich e $\mathrm{R}^{2}$ é o coeficiente de correlação.

$$
\begin{aligned}
& q_{e q}=\frac{q m K_{L} C_{\varepsilon}}{1+K_{L} c_{\varepsilon}} \\
& q_{e}=K_{F} C_{\varepsilon}^{\frac{1}{n}}
\end{aligned}
$$

Os valores de $\mathrm{q}_{\mathrm{m}}$ e $\mathrm{K}_{\mathrm{F}}$ permitem avaliar a capacidade de adsorção, identificando a capacidade do carvão ativado de reter determinado soluto, o que possibilita estimar extensão de seu movimento na fase líquida. Estes coeficientes podem ser entendidos como uma medida da distribuição de equilíbrio entre as fases sólida e líquida. Assim sendo, quanto maior a capacidade adsortiva do material, maiores serão os valores de $\mathrm{q}_{\mathrm{m}}$ e $\mathrm{K}_{\mathrm{F}}$ 
para os modelos de Langmuir e Freundlich, respectivamente.

Foram realizados testes de porcentagem de adsorção individual dos briquetes ativados (PAIB), calculada por meio da Equação 6.

$$
\operatorname{PAIB}(\%)=\frac{\text { ABS }_{\text {Branco }}-\text { ABS }_{\text {Amostra }}}{A B S_{\text {Branco }}} \times 100
$$

em que $\mathrm{ABS}_{\text {Branco }}$ e $\mathrm{ABS}_{\mathrm{Amostra}}$ são as absorbâncias, a $665 \mathrm{~nm}$, para o azul de metileno e $270 \mathrm{~nm}$ para o fenol, do branco e da amostra com $\mathrm{CAB}$, respectivamente.

Cada $\mathrm{CAB}$ foi colocado em uma solução com $100 \mathrm{~mL}$ de contaminante (azul de metileno ou fenol), à concentração de $1000 \mathrm{mg} \mathrm{L}^{-1}$. Os testes têm a finalidade de analisar qual o potencial de adsorção de cada CAB.

\section{9 Índice de resistência ao impacto}

A resistência ao impacto dos materiais ativados foi determinada abandonando, várias vezes, os briquetes de uma altura de $2 \mathrm{~m}$ sobre um piso de concreto até que se quebrasse. O índice de resistência ao impacto (IRI) é calculado dividindo-se o número de vezes que o briquete foi largado contra o piso de concreto (A) pelo número de partes em que o briquete se quebrou (B), conforme a Equação 7. A partir destes dados, o IRI foi calculado [21].

$$
\text { ÍndicederesistênciaaoimpactoIRI }=\frac{\mathrm{A}}{\mathrm{B}} \times 100
$$

\section{RESULTADOS E DISCUSSÃO}

\subsection{Análise elementar $\mathrm{CHN}-\mathrm{O}$}

A Tabela 2 apresenta a composição elementar dos resíduos da madeira de candeia e dos carvões ativados briquetados produzidos (CABs).Quando comparados os carvões ativados e o material precursor, foi observado um aumento no teor de carbono e um aumento na relação $\mathrm{C} / \mathrm{H}$ após os processos de ativação e carbonização. $\mathrm{O}$ aumento da relação $\mathrm{C} / \mathrm{H}$ representa um aumento da aromaticidade do material. $\mathrm{O}$ aumento da temperatura e do tempo de ativação também promove o aumento da relação $\mathrm{C} / \mathrm{H}$. A diminuição no teor de oxigênio e de hidrogênio dos $\mathrm{CABs}$, comparada à do precursor (candeia), ocorre devido à liberação de compostos oxigenados voláteis $[22,23]$.

\begin{tabular}{|c|c|c|c|c|c|}
\hline Amostras & $\mathrm{C}(\%)$ & $\mathrm{H}(\%)$ & $\mathrm{O}^{\mathrm{b}}(\%)$ & $\mathrm{N}(\%)$ & $\mathrm{C} / \mathrm{H}$ \\
\hline Candeia & 50,43 & 5,83 & 41,82 & 1,92 & 8,65 \\
\hline Carbonizado $^{\mathrm{a}}$ & 78,60 & 2,74 & 16,56 & 2,10 & 28,68 \\
\hline CAB700-1 & 81,84 & 2,02 & 14,34 & 1,80 & 40,51 \\
\hline CAB700-3 & 82,86 & 1,61 & 13,65 & 1,88 & 51,4 \\
\hline CAB800-1 & 83,01 & 1,41 & 13,33 & 2,25 & 58,87 \\
\hline CAB800-3 & 82,05 & 1,13 & 15,02 & 1,80 & 72,61 \\
\hline CAB900-1 & 85,11 & 0,92 & 12,18 & 1,79 & 92,51 \\
\hline CAB900-3 & 80,08 & 0,75 & 17,43 & 1,74 & 106,77 \\
\hline
\end{tabular}

Tabela 2: Constituição do resíduo de Candeia

\subsection{Análise da superfície e grupos funcionais}

Os grupos funcionais da superfície dos CABs foram identificados pelo método titulométrico, utilizando-se a técnica de Boehm. As propriedades ácidas da superfície do carvão ativado são causadas pela presença de grupos carboxílicos, lactonas ou lactóis e grupos hidroxila de caráter fenólico. Esses grupos diferem em sua acidez e podem ser diferenciados pela neutralização com soluções de bicarbonato de sódio (NaHCO), carbo- 
BORGES, W.M.S.;ARANTES, A.C.C.; CASTRO, G.M.M.; BIANCHI, M.L.; NOBRE, J.R.C.; RESENDE, E.C.; CASTRO, J.P.; GUERREIRO, M.C. revista Matéria, v.21, n.4, pp. 930 - 942, 2016.

nato de sódio $\left(\mathrm{Na}_{2} \mathrm{CO}_{3}\right)$ e hidróxido de sódio $(\mathrm{NaOH})$ [24]. O total de sítios básicos foi identificado utilizando-se $\mathrm{HCl}$, que reage com todos os grupos básicos.

Na Tabela 3 pode-se observar que o total de sítios básicos tende a aumentar conforme o aumento da temperatura e do tempo de ativação.

Tabela 3: Grupos funcionais e total de sítios ácidos e básicos

\begin{tabular}{|c|c|c|c|c|c|}
\hline \multirow[t]{2}{*}{$\begin{array}{l}\text { Variáveis de } \\
\text { ativação }\end{array}$} & $\begin{array}{l}\text { Total de } \\
\text { sítios bá- } \\
\text { sicos }\end{array}$ & $\begin{array}{l}\text { Total de } \\
\text { sítios áci- } \\
\text { dos }\end{array}$ & $\begin{array}{l}\text { Ác. car- } \\
\text { box. }\end{array}$ & Lactona & Fenóis \\
\hline & \multicolumn{5}{|c|}{$\left(\mathrm{mmolg}^{-1} \mathrm{~A}\right)$} \\
\hline Carbonizado & 1,147 & 1,025 & 0,985 & 0,015 & 0,024 \\
\hline CAB700-1 & 1,261 & 1,057 & 0,915 & 0,043 & 0,097 \\
\hline CAB700-3 & 1,511 & 1,033 & 0,934 & 0,059 & 0,059 \\
\hline CAB800-1 & 1,528 & 1,010 & 0,890 & 0,068 & 0,051 \\
\hline CAB800-3 & 1,581 & 0,991 & 0,899 & 0,042 & 0,048 \\
\hline CAB900-1 & 1,598 & 0,975 & 0,855 & 0,097 & 0,022 \\
\hline CAB900-3 & 1,721 & 0,956 & 0,807 & 0,115 & 0,033 \\
\hline
\end{tabular}

Com o aumento da temperatura e do tempo de ativação, houve uma tendência na diminuição na acidez total. Observou-se diminuição na quantidade de ácido carboxílico na superfície dos carvões ativados. A diminuição na acidez dos materiais ocorre durante a ativação física a altas temperaturas. Há remoção de oxigênio da superfície dos carvões ativados. Sob alta temperatura, acima de $700^{\circ} \mathrm{C}$, o tratamento térmico sob atmosfera inerte pode ser utilizado para remover seletivamente algumas das funcionalidades ácidas da superfície do carvão ativado [25]. As funções que contêm oxigênio são decompostas a $800-1000{ }^{\circ} \mathrm{C}$, aumentando o caráter básico da superfície. A oxidação em fase gasosa dos carvões ativados aumenta, principalmente, a concentração de grupos hidroxila e carbonílicos da superfície [26].

\subsection{Análise da área superficial}

A área superficial dos CABs pode ser relacionada aos valores de burn-off. Observa-se, na Tabela 4, que a área obtida pela adsorção de azul de metileno $\left(\mathrm{S}_{\mathrm{AM}}\right)$ e o índice de iodo aumentam com o valor de burn-off.

Tabela 4:Valores da área superficial dos carvões ativados, obtida por diferentes métodos

\begin{tabular}{c|c|c|c|c|c|c|c}
\hline $\begin{array}{c}\text { Carvão } \\
\text { ativado }\end{array}$ & $\begin{array}{c}\mathrm{S}_{\mathrm{AM}} \\
\left(\mathrm{m}^{2} \mathrm{~g}^{-1}\right)\end{array}$ & $\begin{array}{c}\text { Índice de } \\
\text { iodo } \\
\left(\mathrm{mg} \mathrm{g}^{-1}\right)\end{array}$ & $\begin{array}{c}\text { Área BET } \\
\text { estimada } \\
\left(\mathrm{m}^{2} \mathrm{~g}^{-1}\right)\end{array}$ & $\begin{array}{c}\text { Volume total } \\
\text { de poros } \\
\left(\mathrm{cm}^{3} \mathrm{~g}^{-1}\right)\end{array}$ & $\begin{array}{c}\text { Volume de } \\
\text { microporos } \\
\left(\mathrm{cm}^{3} \mathrm{~g}^{-1}\right)\end{array}$ & $\begin{array}{c}\text { Rend. }(\%) \mathrm{a} \\
\text { partir da car- } \\
\text { bonização }\end{array}$ & $\begin{array}{c}\text { Burn-off } \\
(\%)\end{array}$ \\
\hline CAB700-1 & 11,79 & 37 & - & - & - & 96,55 & 3,4 \\
\hline CAB700-3 & 11,96 & 109 & - & - & - & 90,59 & 9,4 \\
\hline CAB800-1 & 16,31 & 55 & - & - & - & 85,02 & 14,9 \\
\hline CAB800-3 & 25,47 & 194 & - & - & - & 77,58 & 22,4 \\
\hline CAB900-1 & 29,12 & 358 & 364 & 0,20 & 0,10 & 59,62 & 40,3 \\
\hline CAB900-3 & 31,22 & 453 & 409 & 0,21 & 0,13 & 48,21 & 51,7 \\
\hline
\end{tabular}

${ }^{\mathrm{a}}$ Rendimento em relação à massa de material carbonizado

TAER et al. [27] obtiveram resultados semelhantes, quando submeteram pellets a uma variação de temperatura e tempo de ativação, observando-se um aumento na área superficial. 
O azul de metileno é adsorvido em microporos de tamanhos na escala (0,8-2,0nm) e mesoporos (2,0$50 \mathrm{~nm}$ ), e a molécula de iodo é adsorvida em microporos (menores que $0,8 \mathrm{~nm}$ ) [18]. Como os valores de área de $S_{a m}$ não foram significativos para os carvões produzidos, sugere-se que o processo de ativação utilizado não foi capaz de aumentar os poros do material ou produzir microporos grandes ou mesoporos. Uma alternativa para se obter uma área maior com uma quantidades grande de micro e macro poros é a ativação química [5], é visto na literatura que a ativação química do resíduo de madeira provindo da extração do $\alpha$-bisabolol utilizando $\mathrm{ZnCl}_{2}$ e $\mathrm{K}_{2} \mathrm{CO}_{3}$ obtiveram matérias com área superficial BET próxima de $1000 \mathrm{~m}^{2} \mathrm{~g}^{-1}$ [2], porém é importante ressaltar que apesar da obtenção de grandes áreas a ativação química além de ter um custo mais elevado gera resíduos que posteriormente terão que ser tratados, esses fatos torna a ativação física mais interessante pois é mais barata e gera uma quantidade de resíduo menor [6, 7].

Os valores de índice de iodo tiveram um aumento mais expressivo, com o aumento da temperatura e tempo de ativação, sugerindo a formação de microporos menores que $0,8 \mathrm{~nm}$.

$\mathrm{Na}$ ativação de pellets, os processos e as reações químicas que ocorrem na superfície e nas camadas mais internas do material são distintos, assim como o tipo de função química formada é diferente [28]. Nos $\mathrm{CAB}$ de candeia deve ocorrer o mesmo, já que o material não está na forma de pó, e sim, compactado na forma de briquetes.

Só foi possível realizar a estimativa da área BET para os carvões CAB900-1 e 3. O método para se obter os valores de adsorção de azul de metileno foi realizado com o briquete inteiro e o índice de iodo com o briquete macerado. A adsorção do carvão ativado é diferente quando se varia a superfície de contato. Os valores de adsorção obtidos para os CAB700-1, CAB700-3, CAB800-1 e CAB800-3 não se enquadraram no erro do cálculo do software "Structural Characterization of Activated Carbon", sendo possível obter a estimativa da área BET somente com os valores de adsorção dos CAB900-1 e CAB900-3.

Valores de área BET estimada para os materiais CAB900-1 e CAB900-3 estão de acordo com resultados encontrados na literatura. Carvões ativados obtidos a partir de pellets de bagaço e melaço de cana-deaçúcar com ativação física tiveram áreas que variaram de 272 a $455 \mathrm{~m}^{2} \mathrm{~g}^{-1}[29]$.

\subsection{Testes de adsorção}

Todos os carvões ativados produzidos foram testados quanto à capacidade de adsorção das moléculas de azul de metileno e fenol. Os testes tiveram o objetivo de investigar o potencial desses carvões como adsorventes na remediação de efluentes líquidos. Os adsorbatos escolhidos apresentam tamanhos moleculares e propriedades eletrônicas distintas, o que os torna útil para a investigação da adsorção em poros de diferentes dimensões.

Por meio das isotermas de adsorção, apresentadas na Figura 2 é possível observar que o aumento do tempo e da temperatura de ativação promove um aumento na capacidade máxima de adsorção de azul de metileno nos $\mathrm{CAB}$.

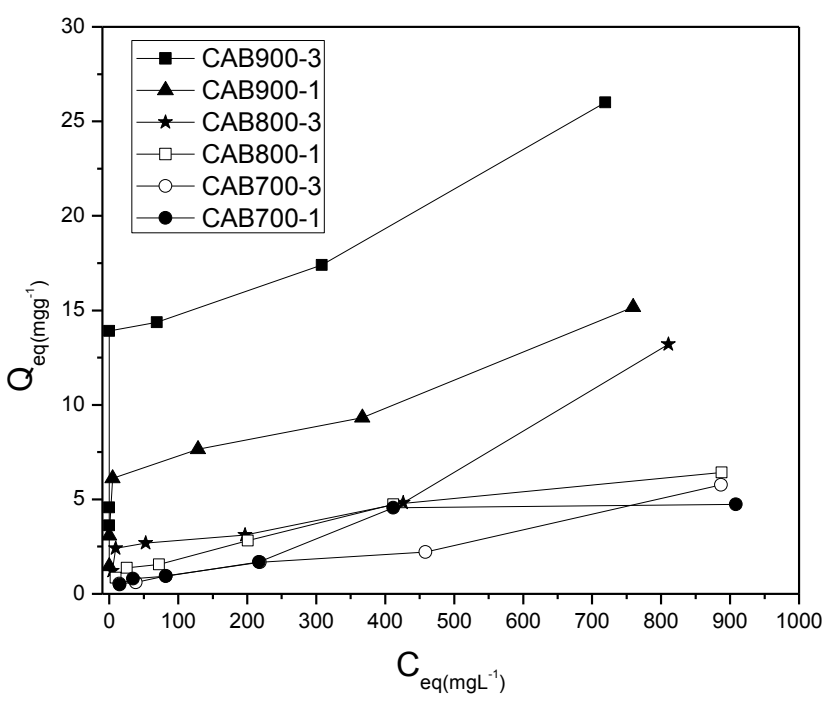

Figura 2: Isotermas de adsorção de AM nos CAB-700-1, CAB700-3, CAB800-1, CAB800-3, CAB900-1 e CAB900-3; (10mg de carvão em $10 \mathrm{~mL}$ de solução de $\mathrm{AM}$ a $10,25,50100,250,500$ e $\left.1.000 \mathrm{mg} \mathrm{L}^{-1}, 24 \mathrm{~h}, 25^{\circ} \mathrm{C}, \mathrm{pH}_{\text {natural }} \approx 6\right)$. 
O melhor resultado obtido foi com o CAB900-3 com um $Q_{e q}=26 \mathrm{mg} \mathrm{g}^{-1}$. Os valores de adsorção de azul de metileno encontrados eram esperados, comparando com os resultados observados na literatura. Na ativação de resíduos sólidos agrícolas para a produção de carvão ativado, foram obtidos valores de adsorção de $12,9 \mathrm{mg} \mathrm{g}^{-1}$ [30]. AYGUN [31] ativou quimicamente granulados de casca de amêndoas, casca de noz, casca de avelã e caroço de damasco e obteve adsorções máximas de $1,33 \mathrm{mg} \mathrm{g}^{-1}, 3,53 \mathrm{mg} \mathrm{g}^{-1}, 8,82 \mathrm{mg} \mathrm{g}^{-1}$ e $4,1 \mathrm{mg}$ $\mathrm{g}^{-1}$, respectivamente.

Na Figura 3 observam-se as porcentagens de remoção de azul de metileno de soluções de concentração de $1000 \mathrm{mg} \mathrm{L}^{-1}$ para cada briquete. Observa-se que o CAB700-1 removeu 10\% do azul de metileno da solução e o CAB900-3 removeu, aproximadamente, 23\%. É observado que mantendo a temperatura de 700 e $900^{\circ} \mathrm{C}$ e variando o tempo de 1 e 3 horas há um pequeno aumento na adsorção de AM, já na temperatura de $800^{\circ} \mathrm{C}$ a variável tempo é significativa no aumento da adsorção. O estudo de tempo e temperatura de ativação é de extrema importância na viabilidade energética, grandes temperaturas demandam um custo energético alto e dependendo da finalidade que o material irá ser empregado a ativação se torna inviável.

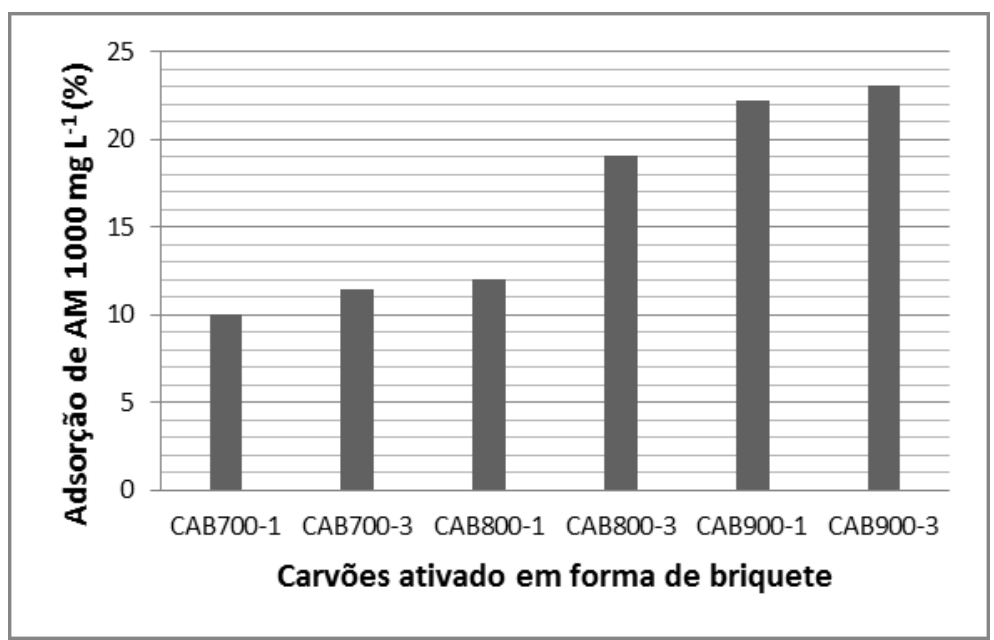

Figura 3: Porcentagem de adsorção de azul de metileno pelos carvões ativados briquetados em solução de concentração $1000 \mathrm{mg} \mathrm{L}^{-1}, \mathrm{pH}_{\text {natural }} \approx 6$.

Os valores de $\mathrm{q}_{\mathrm{m}}$ e $\mathrm{K}_{\mathrm{F}}$ permitem avaliar a capacidade de adsorção identificando a capacidade do CAB de reter determinado soluto, o que possibilita a estimativa da extensão de seu movimento na fase líquida. Estes coeficientes podem ser entendidos como uma medida da distribuição de equilíbrio entre as fases sólida e líquida. Assim sendo, quanto maior a capacidade adsortiva do material, maiores serão os valores de $\mathrm{q}_{\mathrm{m}}$ e $\mathrm{K}_{\mathrm{F}}$ para os respectivos modelos de Langmuir e Freundlich.

De acordo com os dados experimentais apresentados na Tabela 5, os resultados obtidos para os CABs apresentaram melhores ajustes para o modelo de Freundlich, sugerindo que o processo de adsorção ocorre em uma superfície heterogênea, considerando que os sítios de adsorções têm capacidade de adsorções diferentes e que apresentam variações em função da cobertura da superfície [32].

O CAB900-3 apresenta na isoterma de Lagmuir um valor de $\mathrm{K}_{\mathrm{L}}$ superior aos outros CABs, pode-se concluir que o aumento de tempo de ativação apresenta uma modificação nos sítios de ligação obtendo uma maior energia de adsorção, apesar do CAB900-1 apresentar um valor próximo de qm do CAB900-3 o $\mathrm{K}_{\mathrm{L}}$ do CAB900-1 sugere que o material possua sitos com energia de adsorção menor.

Tabela 5: Parâmetros de Langmuir e Freundlich para adsorção de azul de metileno nos diferentes carvões ativado.

\begin{tabular}{l|c|l|l}
\hline \multicolumn{3}{l}{ Azul de metileno } \\
\hline \multirow{2}{*}{ Materiais } & \multicolumn{3}{|c}{ Langmuir } \\
\cline { 2 - 4 } & $\mathrm{qm}\left(\mathrm{mgg}^{-1}\right)$ & $\mathrm{K}_{\mathrm{L}}\left(\mathrm{Lmg}^{-1}\right)$ & $\mathrm{R}^{2}$ \\
\hline CAB700-1 & 6,1 & 0,002 & 0,88 \\
\hline CAB700-3 & 6,2 & 0,002 & 0,83 \\
\hline CAB800-1 & 8,4 & 0,002 & 0,95 \\
\hline
\end{tabular}


BORGES, W.M.S.;ARANTES, A.C.C.; CASTRO, G.M.M.; BIANCHI, M.L.; NOBRE, J.R.C.; RESENDE, E.C.; CASTRO, J.P.; GUERREIRO, M.C. revista Matéria, v.21, n.4, pp. 930 - 942, 2016.

\begin{tabular}{c|l|l|l}
\hline CAB800-3 & 13,2 & 0,003 & 0,73 \\
\hline CAB900-1 & 16,0 & 0,006 & 0,64 \\
\hline \multirow{2}{*}{ CAB900-3 } & 16,1 & 0,45 & 0,82 \\
\hline \multirow{2}{*}{ Materiais } & \multicolumn{2}{|l}{ Freundlich } & $\mathrm{R}^{2}$ \\
\cline { 2 - 4 } & $1 / \mathrm{n}$ & $\mathrm{K}_{\mathrm{F}}\left(\mathrm{mgL}^{-1}\right)\left(\mathrm{Lmg}^{-1}\right)^{1 / \mathrm{n}}$ & 0,89 \\
\hline CAB700-1 & 0,56 & 0,100 & 0,95 \\
\hline CAB700-3 & 0,86 & 0,015 & 0,95 \\
\hline CAB800-1 & 0,85 & 0,016 & 0,88 \\
\hline CAB800-3 & 1,02 & 0,013 & 0,80 \\
\hline CAB900-1 & 0,22 & 2,890 & 0,98 \\
\hline CAB900-3 & 0,09 & 9,400 & \\
\hline
\end{tabular}

Observa-se na Figura 4 que os CABs tiveram um aumento na capacidade máxima de adsorção de fenol, quando houve aumento na temperatura e no tempo de ativação. Isso ocorreu porque essas variações provocam aumento da área superficial, do volume de poros e da basicidade dos carvões, como apresentado na Tabela 4. O CAB900-3 apresentou o maior valor de adsorção do fenol, já que é o mais básico e tem grande volume de microporos, em que o adsorbato pode ser adsorvido.

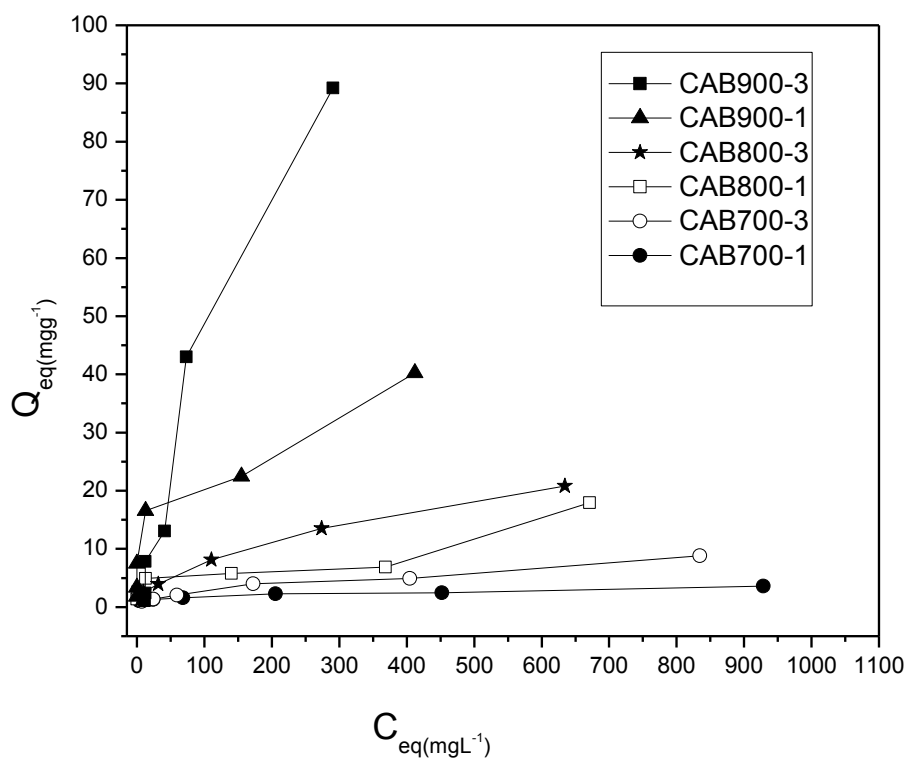

Figura 4: Isotermas de adsorção de fenol nos CAB-700-1, CAB700-3, CAB800-1, CAB800-3, CAB900-1e CAB900-3; (10 mg de carvão em $10 \mathrm{~mL}$ de solução de fenol a 10, 25, $50100,250,500$ e $1.000 \mathrm{mg} \mathrm{L}^{-1}, 24 \mathrm{~h}, 25^{\circ} \mathrm{C}, \mathrm{C} \mathrm{pH}_{\text {natural }} \approx 6$ ).

A adsorção do fenol nos carvões ativados está relacionada com as características da área, porosidade e as propriedades da superfície. O fenol se liga na superfície do carvão ativado por meio de interações entre os elétrons do sistema- $\pi$, ou seja, os elétrons- $\pi$ do anel aromático do fenol interagem por forças de London ao sistema- $\pi$ das camadas grafíticas do carvão [14]. Essa interação ocorre paralelamente ao plano basal do carvão ativado, ou com formação de um complexo doador-receptor. Nesse tipo de interação, os grupos superficiais, em especial a carbonila, interagem com o grupo $\mathrm{OH}$ do fenol. A ligação ocorre com o fenol perpendicular ao plano basal das camadas grafíticas. Esse tipo de ligação tem sido apontado como a principal causa da irreversibilidade do processo de adsorção do fenol em carvão ativado [14, 33].

Os dados de adsorção do fenol nos diferentes carvões foram ajustados segundo os modelos de Langmuir e Freundlich, tendo o modelo de Freundlich fornecido os melhores resultados de $\mathrm{R}^{2}$ para todos dos CABs. Os resultados dos ajustes estão resumidos na Tabela 6. 
BORGES, W.M.S.;ARANTES, A.C.C.; CASTRO, G.M.M.; BIANCHI, M.L.; NOBRE, J.R.C.; RESENDE, E.C.; CASTRO, J.P.; GUERREIRO, M.C. revista Matéria, v.21, n.4, pp. 930 - 942, 2016.

Tabela 6: Parâmetros de Langmuir e Freundlich para adsorção de fenol nos diferentes carvões ativado.

\begin{tabular}{l|c|c|c}
\hline \multirow{2}{*}{ Menol } & \multicolumn{3}{|c}{ Langmuir } \\
\cline { 2 - 4 } & $\mathrm{qm}\left(\mathrm{mgg}^{-1}\right)$ & $\mathrm{K} 1\left(\mathrm{Lmg}^{-1}\right)$ & $\mathrm{R}^{2}$ \\
\hline CAB700-1 & 3,10 & 0,020 & 0,71 \\
\hline CAB700-3 & 9,16 & 0,002 & 0,82 \\
\hline CAB800-1 & 17,30 & 0,002 & 0,65 \\
\hline CAB800-3 & 23,05 & 0,005 & 0,95 \\
\hline CAB900-1 & 42,80 & 0,010 & 0,81 \\
\hline CAB900-3 & 98,20 & 0,009 & 0,9 \\
\hline \multirow{2}{*}{ Materiais } & & Freundlich & $\mathrm{R}^{2}$ \\
\cline { 2 - 4 } & $1 / \mathrm{n}$ & $\mathrm{KF}\left(\mathrm{mgL}^{-1}\right)\left(\mathrm{Lmg}^{-1}\right)^{1 / \mathrm{n}}$ & 0,94 \\
\hline CA700-1 & 0,25 & 0,59 & 0,98 \\
\hline CA700-3 & 0,53 & 0,23 & 0,78 \\
\hline CA800-1 & 0,69 & 0,16 & 0,99 \\
\hline CA800-3 & 0,53 & 0,65 & 0,94 \\
\hline CA900-1 & 0,36 & 4,35 & 0,96 \\
\hline CAB900-3 & 0,78 & 1,07 &
\end{tabular}

Avaliando a porcentagem de remoção de fenol de uma solução de $1000 \mathrm{mg} \mathrm{L}^{-1}$ pelos CABs, apresentado na Figura 5, observa-se que o CAB700-1 adsorveu somente 9,0\%; já o CAB900-3 adsorveu, aproximadamente, $73 \%$. O aumento de temperatura e tempo de ativação causa uma mudança nas características do material como o aumento na área superficial Tabela 4 e diminuição dos grupos ácidos Tabela 3, resultando em um aumento significativo na adsorção.

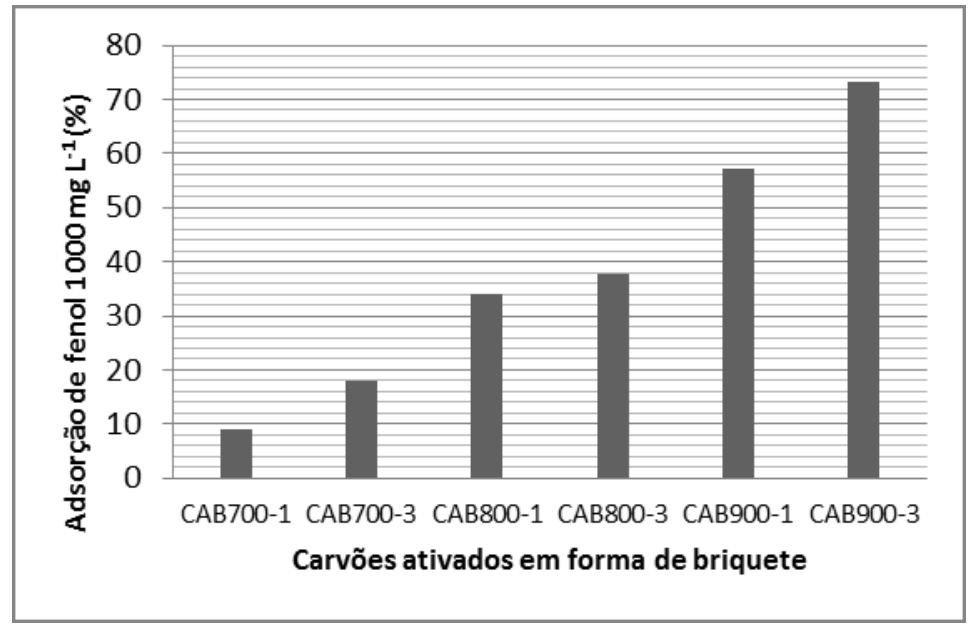

Figura 5: Porcentagem de adsorção do fenol pelos carvões ativados briquetados em solução de concentração $1000 \mathrm{mg} \mathrm{L}^{-1}$.

Como os carvões ativados estão na forma de briquetes, o interior do material teve um menor contato com o agente oxidante, ocorrendo uma ativação expressiva na superfície do briquete [28]. Além disso, a área dos CABs em contato com a solução a ser descontaminada é menor, se comparada à área de uma mesma massa de carvão ativado em pó. Porém, os resultados mostram que o CAB é capaz de adsorver fenol, além de ser facilmente removido da solução após a adsorção. Dessa maneira, os CABs têm potencial para serem utilizados como adsorvente.

\section{5 Índice de Resistência ao Impacto (IRI)}

O índice de resistência mecânica é uma característica importante do briquete para sua utilização. CABs com 
pouca resistência física se quebram e são inviáveis. Somente IRI maiores que 50 são aceitos pela indústria [34]. Se compararmos esse valor com os verificados pelos CABs produzidos, de acordo com a Tabela 7, podermos concluir que a resistência exigida pelas indústria foi superada em $14 \%$ para o CAB900-3 e de até $300 \%$ para o CAB700-1.

Observa-se que, com o aumento do tempo e da temperatura de ativação, tem-se uma diminuição do IRI. Isso porque, em condições mais drásticas de ativação, o agente ativante $\left(\mathrm{CO}_{2(\mathrm{~g})}\right)$ provoca uma maior oxidação do material e, assim, diminuindo o IRI, corroborando os resultados observados na literatura [35].

Tabela 7: Índice de resistência de impacto.

\begin{tabular}{l|l}
\hline Amostras & IRI \\
\hline CAB700-1 & 150 \\
\hline CAB700-3 & 120 \\
\hline CAB800-1 & 100 \\
\hline CAB800-3 & 100 \\
\hline CAB900-1 & 88 \\
\hline CAB900-3 & 57 \\
\hline
\end{tabular}

O CAB900-3 obteve os melhores valores para a adsorção de fenol e azul de metileno. E o menor valor de IRI (57), apesar do CAB900-3 apresentar a menor resistência obteve um valor superior ao exigido pela indústria. DEIANA et al. [8] obteve carvões ativados em forma de briquetes utilizando um mosto de uva como lingante, o trabalho apresenta materiais com IRI variando de 25 a 350. É importante salientar que os CABs não utilizaram nenhum tipo de ligante. O resíduo de madeira provindo da candeia mostrou-se não precisar de nenhum ligante apresentando IRI satisfatórios.

\section{CONCLUSÃO}

A partir dos resultados obtidos no presente trabalho pode-se verificar que o resíduo madeireiro de candeia é uma matéria-prima de grande potencial para a produção de carvão ativado em forma de briquete. $\mathrm{O}$ material precursor passou pelo processo de briquetagem, carbonização, ativação e apresentou IRI satisfatório, não exigindo a utilização de compostos para auxiliarem na compactação.

As variações na temperatura e no tempo de ativação têm influência na formação dos grupos químicos presentes na superfície. Com o aumento da temperatura e do tempo de ativação, observa-se o aumento da área superficial, do volume de poros, da basicidade e da capacidade de adsorção. Por outro lado, tem-se a diminuição do rendimento do carvão e do IRI.

O carvão ativado de briquete mostrou ser um bom adsorvente para azul de metileno e fenol, adsorvendo $73 \%$ de fenol e $23 \%$ de azul de metileno em concentrações de $1.000 \mathrm{mg} \mathrm{L}^{-1}$ em ensaio do tipo batelada. Os melhores CABs apresentaram na suas isotermas capacidade máxima de adsorção de AM de $16,1 \mathrm{mg} \mathrm{g}^{-1}$ com um $\mathrm{K}_{\mathrm{F}}$ de $0.45 \mathrm{~L} \mathrm{mg}^{-1}$ e um $\mathrm{K}_{\mathrm{L}}$ de $9.04\left(\mathrm{mgL}^{-1}\right)\left(\mathrm{Lmg}^{-1}\right)^{1 / \mathrm{n}}$, para as curvas de adsorção de fenol obteve-se uma capacidade máxima de adsorção de $98,20 \mathrm{mg} \mathrm{g}^{-1}$ com um $\mathrm{K}_{\mathrm{F}}$ de $0,009 \mathrm{~L} \mathrm{mg}^{-1}$ e um $\mathrm{K}_{\mathrm{L}}$ de $1,07\left(\mathrm{mgL}^{-1}\right)$ $\left(\mathrm{Lmg}^{-1}\right)^{1 / \mathrm{n}}$.

Essas características tornam os CABs de candeia uma alternativa interessante para a produção de carvão ativado em forma de bríquete.

\section{BIBLIOGRAFIA}

[1] ROSIMEIRE, C. S., LOURIVAL, M. M., FÁBIO, A. M., et. al., “Aproveitamento de resíduos da madeira de candeia (Eremanthuserythropappus) para produção de painéis cimento-madeira”, Cerne, v. 14, n. 3, pp. 241-250, Jan. 2008.

[2] EliAne, C. R., PAUliZA, H. R., MÁRIO, C. G., et al., "Production and characterization of activated carbons from waste candeia (EremanthusErythropappus) and their aplication on organic compounds adsorption produção", Revista Colombiana de Quimima, v. 39, n. 1, pp. 111-120, Apr. 2010.

[3] HASAN, S., FUAT. G.,YUNUS. O., "Conversion of grape industrial processing waste to activated carbon sorbent and its performance in cationic and anionic dyes adsorption", Journal Of Cleaner Production, v. 93, n. 2, pp. 1-10, Jan. 2015. 
BORGES, W.M.S.;ARANTES, A.C.C.; CASTRO, G.M.M.; BIANCHI, M.L.; NOBRE, J.R.C.; RESENDE, E.C.; CASTRO, J.P.; GUERREIRO, M.C. revista Matéria, v.21, n.4, pp. 930 - 942, 2016.

[4] JOÃO, M. V. N., CARLOS. E. C. L., P.J.M. C., et al., "Production of activated carbons from almond shell," Fuel Processing Technology, v. 92, n. 2, pp. 234-240, Feb. 2011.

[5] MOLINA-SABIO, M., RODRIGUEZ-REINOSO. F., "Role of chemical activation in the development of carbon porosity", Colloids Surfaces A: Physicochemical and Enginneering Aspects, v. 241, n. 1-3, pp. 1525, Jul. 2004.

[6] MARÍA, S. C., CARLOS. A. P., LEIRE. Z., et al., "A comparison of physical activation of carbon xerogels with carbon dioxide with chemical activation using hydroxides", Carbon, v. 48, n. iii, pp. 3157-3168, May 2010.

[7] MACIÁ-AGULlÓ, J. A., MOORE. B. C., CAZORLA-AMORÓS. D., et al., "Activation of coal tar pitch carbon fibres: Physical activation vs. chemical activation", Carbon, v. 42, pp. 1361-1364, Dec. 2004.

[8] DEIANA, A. C., GRANADOS. L. M., PETKOVIS. M.F., et al., "Use of grape must as a binder to obtain activated carbon briquettes", Brazilian Journal Chemical Engineering, v. 21, n. 04, pp. 585-591, Dec. 2004.

[9] ZHIQUAN, Q. Y., JOHN. C. M., EDWARD. D. T., "Preparation of high activity, high density activated carbon with activatable binder", 1996.

[10] LONGJIAN, C., LI. X., LUJIA. H., "Renewable energy from agro-residues in China: Solid biofuels and biomass briquetting technology", Renewable Sustainable Energy Reviews, v. 13, n. 9, pp. 2689-2695, Dec. 2009.

[11] NALLADURAI, K., VANCE. M. R., "Factors affecting strength and durability of densified biomass products", Biomass and Bioenergy, v. 33, n. 3, pp. 337-359, Mar. 2009.

[12] YUAN, G., QIN-YAN. Y., YUAN-YUAN. S., et al., "Optimization of high surface area activated carbon production from Enteromorphaprolifra with low-dose activating agent", Fuel Processing Technology, v. 132, pp. 180-187, Jan. 2015.

[13] BOEHM, H. P., "Some aspects of the surface chemistry of carbon blacks and other carbons", Carbon, v. 32, n. 5, pp. 759-769, Jan. 1994.

[14] VIVIANE, V. S. G., JOÃO. P. M., PATRÍCIA. B. M., et al., "Adsorção de fenol sobre carvão ativado em meio alcalino", Química Nova, v. 29, n. 6, pp. 1226-1232, Jun. 2006.

[15] ALICIA, M. O., SARAH. L. G., KATELYN. R. H., et al., "Standardization of the Boehm titration: Part II. Method of agitation, effect of filtering and dilute titrant", Carbon, v. 48, n. 12, pp. 3313-3322, May 2010.

[16] SARAH, L. G., KIM. D. T., ALICIA. M. O., et al., "Standardization of the Boehm titration. Part I. CO2 expulsion and endpoint determination", Carbon, v. 48, pp. 1252-1261, Dec. 2010.

[17] TERESA, J. BANDOSZ, Activated Carbon Surfaces in Environmental Remediation, 1st ed., v. 7. New York, Elsevier, 2006.

[18] CLEITON, A. N., MÁRIO. C. G., "Estimation of Surface Area and Pore Volume of Activates Carbons by Methylene Blue and Iodine Numbers", Química Nova, v. 34, n. 3, pp. 472-476, Jan. 2011.

[19] AMERICAN SOCIETY FOR TESTING AND MATERIALS., "D4607 - 94 Standard Test Method for Determination of Iodine Number of Activated Carbon", 2011.

[20] STAVROPOUlOS, G. G., ZABANIOTOU. A. A., "Production and characterization of activated carbons from olive-seed waste residue", Microporous Mesoporous Materials, v. 82, n. 1, pp. 79-85, Mar. 2005.

[21] RICHARDS, S. R., "Physical testing of fuel briquettes", Fuel Processing Technology, v. 25, pp. 89100, Sep. 1990.

[22] GOUTAM, C., DOUGLAS. G. M., NARENDRA. N. B., et al. "Preparation and characterization of chars and activated carbons from Saskatchewan lignite", Fuel Processing Technology, v. 87, n. 11, pp. 9971006, Jul. 2006.

[23] GABRIELA, M. C., ANELISE. L. A. D., MARIA. L. B., et al., "Use of sawdust Eucalyptus sp. in the preparation of activated carbons", Ciência e Agrotecnologia, v. 36, n. 1, pp. 69-77, Feb. 2012.

[24] BOEHM, H. P., "Surface oxide on carbon and their analysis: a critical assessment", Carbon, v. 151, n. 3, pp. 417-30, Mar. 2007.

[25] SEYED, A. D., TANJU. K., "Adsorption of oxygen by heat-treated granular and fibrous activated carbons", Journal Colloid and Interface Science, v. 274, n. 1, pp. 1-8, Jan. 2004.

[26] Figueiredo, J. L., PEREIRA. M. F. R., FREITAS. M. M. A., et al., "Modification of the surface chemistry of activated carbons", Carbon, v. 37, n. 9, pp. 1379-1389, Dec. 1999. 
[27] ERMAN, T., MOHAMAD. D., IBRAHIM. A. T., et al., "Physical, electrochemical and supercapacitive properties of activated carbon pellets from pre-carbonized rubber wood sawdust by $\mathrm{CO} 2$ activation", Current Applied Physics, v. 10, n. 4, pp. 1071-1075, Jan. 2010.

[28] LUIZ, C. M. C., MARIA. H. A., KARIM. S., et al., "High surface area functionalized carbon briquettes: A novel Adsorbent for contaminants from water", Journal of th Brazilian Chemical Society, v. 16, n. 5, pp. 899-902, Sep. 2005.

[29] GILBERTO, C. G., ELISABETE. S. M., NEHEMIAS. C. P., et al., "Produção de carvão ativado a partir de bagaço e melaço de cana”, Acta Scientiarum Technology, v. 28, pp. 21-27, Jun. 2006.

[30] FAWZI, B., SAMEER. A., LEEMA. A., "Evaluation of the use of raw and activated date pits as potential adsorbents for dye containing waters", Process Biochemistry, v. 39, n. 2, pp. 193-202, Feb. 2003.

[31] AYGUN, A, YENISOY-KARAKAS. S., DUMAN. I., "Production of granular activated carbon from fruit stones and nutshells and evaluation of their physical, chemical and adsorption properties", Microporous Mesoporous Materials, v. 66, n. 2-3, pp. 189-195, Dec. 2003.

[32] ALINE, D., MARIA. C. T. L., CLÓVIS. A. R.,"Adsorption of congo red dye by hydrophobic Ocarboxymethyl chitosan derivatives”, Química Nova, v. 34, n. 5, pp. 764-770, Mar. 2011.

[33] DABROWSKI, A., PODKOSCIELNY. P., HUBICKI. Z., et al., "Adsorption of phenolic compounds by activated carbon - A critical review”, Chemosphere, v. 58, n. 8, pp. 1049-1070, Sep. 2005.

[34] RUBIO, B., IZQUIERDO. M. T., SEGURA. E., "Effect of binder addition on the mechanical and physicochemical properties of low rank coal char briquettes”, Carbon, v. 37, n. 11, pp. 1833-1841, Jan. 1999.

[35] ALEJANDRO, A., NATALIA. M., NÉSTOR. T., et al., "Activated carbon briquettes from biomass materials", Bioresource Technology, v. 98, n. 8, pp. 1635-41, May 2007. 